\title{
Analysis of Variance in an Unbalanced Two-Way Mixed Effect Interactive Model
}

\author{
F. C. Eze, E. U. Nwankwo \\ Department of Statistics, Nnamdi-Azikiwe University, Awka, Nigeria \\ Email: ezefcc@yahoo.com
}

Received 9 February 2016; accepted 23 April 2016; published 26 April 2016

Copyright (C) 2016 by authors and Scientific Research Publishing Inc.

This work is licensed under the Creative Commons Attribution International License (CC BY).

http://creativecommons.org/licenses/by/4.0/

(c) (i) Dpen Access

\begin{abstract}
The expected mean squares for unbalanced mixed effect interactive model were derived using Brute Force Method. From the expected mean squares, there are no obvious denominators for testing for the main effects when the factors are mixed. An expression for F-test for testing for the main effects was derived which was proved to be unbiased.
\end{abstract}

\section{Keywords}

Mixed Model, Expected Mean Squares, Unbalanced Data

\section{Introduction}

The problem with unbalanced fixed effect interactive model is associated with the appropriate F-test for testing for the main effects when interactions are present. The paper by [1] worked on application of mixed-effects model for exposure assessment by re-analyzing three data sets from published surveys with repeated exposure measurements. The relative contributions of particular characteristics affecting exposure levels were assessed as in a multiple regression model, while controlling for the correlation between repeated measurements.

In [2], they studied a mixed-effects nonlinear regression for unbalanced repeated measures by estimating and comparing the parameters of a generalized mixed-effects nonlinear regression model. The results are applied to in vitro data on the water transport kinetics of hemodialyzers used in the treatment of patients with chronic renal failure.

Similarly, [3] developed a method for deriving exact tests for variance components in some unbalanced mixed linear models. The derivation was based on a new kind of preliminary orthogonal transformation and a subsequent resampling procedure. The resulting tests are based on mutually independent sums of squares which, under null hypothesis, are distributed as scalar multiples of chi-square variates.

Also [4] derived exact tests for testing hypotheses concerning the variance components of the main effects in an unbalanced random two-way crossed classification with interaction model. The tests are based on four sums 
of squares that are distributed independently as scalar multiple chi-square variates. These sums of squares can be used to find an exact test concerning the interaction variance components.

However, [5] considered the Two-Way ANOVA model with unequal cell frequencies without the assumption of equal error variances. They used generalized approach to finding p-values, classical F-tests for no interaction effects and equal main effects are extended under heteroscedasticity. The generalized F-test they developed in their article can be utilized in significance testing or in fixed level testing under the Neyman-Pearson theory. The problem in their work is that, the assumption of ANOVA was violated.

Analysis of variance is straightforward when an experimental design is balanced, but unequal cell sizes affect the computation of means, hypotheses tested and F-statistics [6].

Several solutions have been proposed for the analysis of unbalanced data. Solutions have focused on forcing the unbalanced data to be balanced. Suggestions include imputing cell means as additional data points into the smaller cells.

Given the model

$$
y_{i j k}=\mu+\alpha_{i}+\beta_{j}+\lambda_{i j}+e_{i j k}\left\{\begin{array}{l}
i=1,2, \cdots, p \\
j=1,2, \cdots, q \\
k=1,2, \cdots, n_{i j}
\end{array}\right.
$$

$y_{i j k}$ is the $k$ th observation in $i j$ th cell;

$\mu$ is the overall mean effects;

$\alpha_{i}$ is the average effects of factor A;

$\beta_{j}$ is the average effects of factor $\mathrm{B}$;

$\lambda_{i j}$ is the effects of the interaction between factor A and factor B;

$e_{i j k}$ is a random error components and;

$n_{i j}$ is the number of observations per cell.

To derive the expected mean squares for Equation (1), [7] derived the expected mean square for factor A when factor $\mathrm{A}$ is fixed and factor $\mathrm{B}$ is random as

$$
E M S_{A}=\left[\sum_{i=1}^{p} n_{i} \alpha_{i}^{2}-\frac{\left(\sum_{i=1}^{p} n_{i} \alpha_{i}\right)^{2}}{n_{.}}\right]+\left[\sum_{i=1}^{p} \frac{\sum_{j=1}^{q} n_{i j}^{2}}{n_{i .}}-\frac{\sum_{i=1}^{q} n_{. i}^{2}}{n_{. .}}\right] \sigma_{\beta}^{2}+\left[\sum_{i=1}^{p} \frac{\sum_{j=1}^{q} n_{i j}^{2}}{n_{i .}}-\frac{\sum_{i j}^{p q} n_{i j}^{2}}{n_{. .}}\right] \sigma_{\lambda}^{2}+(p-1) \sigma_{e}^{2} .
$$

According to him, in mixed models, the expected values of the sums of squares contain functions of the fixed effects that cannot be eliminated by considering linear combinations of the sums of squares. He suggested two obvious ways of overcoming the difficulties associated with unbalanced mixed effect data. The first is to ignore the fixed effects and eliminate them from the model. What remains is a random model for which the F-test can be determined. The second possibility is to assume the fixed effects as random and therefore assume the entire model as random effect models. These suggestions are in fact unsatisfactory.

\section{Expected Mean Squares}

\subsection{Two-Way Unbalanced Random Effect Model}

From Equation (1) above, [8] derived the expected mean squares for unbalanced two-way interactive random model.

They derive the expected mean squares for Equation (1) as shown in Table 1.

Where

$$
\begin{gathered}
n_{i j}=\sum_{i=1}^{p} N_{i}=\sum_{j=1}^{q} N_{j}=N \\
k_{\alpha}=\frac{N-N^{-1} \sum_{i} N_{i}^{2}}{p-1} ;
\end{gathered}
$$


Table 1. ANOVA table for unbalanced two-way interactive random model.

\begin{tabular}{ccccc}
\hline S.V & d.f & SS & MS & Expected mean squares \\
\hline Factor A & $p-1$ & $\mathrm{SS}_{\mathrm{A}}$ & $\mathrm{MS}_{\mathrm{A}}$ & $\sigma_{e}^{2}+k_{\alpha} \sigma_{\alpha}^{2}+k_{1} \sigma_{\lambda}^{2}$ \\
Factor B & $q-1$ & $\mathrm{SS}_{\mathrm{B}}$ & $\mathrm{MS}_{\mathrm{B}}$ & $\sigma_{e}^{2}+k_{\beta} \sigma_{\beta}^{2}+k_{2} \sigma_{\lambda}^{2}$ \\
AxB & $(p-1)(q-1)$ & $\mathrm{SS}_{\lambda}$ & $\mathrm{MS}_{\lambda}$ & $\sigma_{e}^{2}+k_{3} \sigma_{\lambda}^{2}$ \\
Error & $N-p q$ & $\mathrm{SS}_{e}$ & $\mathrm{MS}_{e}$ & $\sigma_{e}^{2}$ \\
Total & $N-1$ & $\mathrm{SS}_{\mathrm{T}}$ & & \\
\hline
\end{tabular}

$$
\begin{gathered}
k_{\beta}=\frac{N-N^{-1} \sum_{j} N_{j}^{2}}{q-1} \\
k_{1}=\frac{\sum_{i} N_{i}^{-1} \sum_{j} n_{i j}^{2}-N^{-1} \sum_{i j} n_{i j}^{2}}{p-1} \\
k_{2}=\frac{\sum_{j} N_{j}^{-1} \sum_{i} n_{i j}^{2}-N^{-1} \sum_{i j} n_{i j}^{2}}{q-1} \\
k_{3}=\frac{N-\sum_{i} N_{i}^{-1} \sum_{j} n_{i j}^{2}-\sum_{j} N_{j}^{-1} \sum_{i} n_{i j}^{2}-N^{-1} \sum_{i j} n_{i j}^{2}+2 \sum_{i j} n_{i j}^{3} N_{i}^{-1} N_{j}^{-1}}{(p-1)(q-1)}
\end{gathered}
$$

and

$$
\begin{gathered}
\sigma_{a}^{2}=E\left(\bar{y}_{i . .}-\bar{y}_{\ldots . .}\right)^{2}=\frac{\sum_{i}\left(\bar{y}_{i . . .}-\bar{y}_{\ldots .}\right)^{2}}{n} ; \\
\sigma_{\beta}^{2}=E\left(\bar{y}_{. j .}-\bar{y}_{\ldots . .}\right)^{2}=\frac{\sum_{j}\left(\bar{y}_{. j .}-\bar{y}_{\ldots . .}\right)^{2}}{n} \\
\sigma_{e}^{2}=E\left(y_{i j k}-\bar{y}_{i j .}\right)^{2}=\frac{\sum_{i j k}\left(y_{i j k}-\bar{y}_{i j .}\right)^{2}}{n} .
\end{gathered}
$$

From Table 1, they found a linear combination of the mean squares with the expected mean squares and derived an expression for testing for

$$
H_{0}: \sigma_{\alpha}^{2}=0 ; H_{0}: \sigma_{\beta}^{2}=0 ; \text { and } H_{0}: \sigma_{\lambda}^{2}=0 .
$$

With the corresponding F-ratios as

$$
\begin{aligned}
& \text { For } H_{01}: \sigma_{\alpha}^{2}=0, F_{f_{\alpha}, f_{\theta_{1}}}^{\alpha}=\frac{M S_{\alpha}}{M S_{\theta_{1}^{\prime}}} \\
& \text { For } H_{02}: \sigma_{\beta}^{2}=0, F_{f_{\beta}, f_{\theta_{2}}}^{\alpha}=\frac{M S_{\beta}}{M S_{\theta_{2}^{\prime}}} \\
& \text { For } H_{03}: \sigma_{\lambda}^{2}=0, F_{f_{\lambda}, f_{e}}=\frac{M S_{\lambda}}{M S_{e}}
\end{aligned}
$$

where $f_{\alpha}, f_{\beta}, f_{\lambda}$ and $f_{e}$ are degrees of for factor $\mathrm{A}$, factor $\mathrm{B}$, the interaction between factor $\mathrm{A}$ and factor $\mathrm{B}$ and 
error components respectively.

And

$$
\begin{gathered}
M S_{\theta_{1}^{\prime}}=\left(1-\theta_{1}\right) M S_{e}+\theta_{1} M S_{\lambda} ; \theta_{1}=\frac{k_{1}}{k_{3}} \\
M S_{\theta_{2}^{\prime}}=\left(1-\theta_{2}\right) M S_{e}+\theta_{2} M S_{\lambda} ; \theta_{2}=\frac{k_{2}}{k_{3}} \\
f_{\theta_{1}}=\frac{\left(M S_{\theta_{1}^{\prime}}\right)^{2}}{\left(1-\theta_{1}\right)^{2} \frac{\left(M S_{e}\right)^{2}}{f_{e}}+\theta_{1}^{2} \frac{\left(M S_{\lambda}\right)^{2}}{f_{\lambda}}} \\
f_{\theta_{2}}=\frac{\left(M S_{\theta_{2}^{\prime}}\right)^{2}}{\left(1-\theta_{2}\right)^{2} \frac{\left(M S_{e}\right)^{2}}{f_{e}}+\theta_{2}^{2} \frac{\left(M S_{\lambda}\right)^{2}}{f_{\lambda}} .}
\end{gathered}
$$

The sums of squares for factor $\mathrm{A}$, factor $\mathrm{B}$, the interaction between factor $\mathrm{A}$ and factor and the error terms are given by

$$
\begin{gathered}
S S_{A}=\sum_{i} N_{i}\left(Y_{i . .}-\bar{Y}_{\ldots}\right)^{2} \\
S S_{B}=\sum_{j} N_{j}\left(Y_{. j .}-\bar{Y}_{\ldots .}\right)^{2} \\
S S_{\lambda}=\sum_{i j} n_{i j}\left(\bar{Y}_{i j .}-\bar{Y}_{i . .}-\bar{Y}_{. j .}+\bar{Y}_{\ldots}\right)^{2} \\
S S_{e}=\sum_{i j k}\left(Y_{i j k}-\bar{Y}_{i j .}\right)^{2} .
\end{gathered}
$$

The expression for testing for the presence of interaction from Table 1 is

$$
\frac{M S_{\lambda}}{M S_{e}} \text {. }
$$

\subsection{Two-Way Unbalanced Mixed Effect Model}

From Equation (1), if factor $\mathrm{A}$ is fixed and factor B is random

$$
\begin{gathered}
\sum_{i} \alpha_{i}=\sum_{i} \lambda_{i j}=0 \\
\beta_{j} \sim N\left(0, \sigma_{\beta}^{2}\right)
\end{gathered}
$$

and

$$
e_{i j k} \sim N\left(0, \sigma_{e}^{2}\right)
$$

Similarly, if factor A is random and factor B is fixed

$$
\begin{gathered}
\sum_{j} \beta_{j}=\sum_{j} \lambda_{i j}=0 \\
\alpha_{i} \sim N\left(0, \sigma_{\alpha}^{2}\right)
\end{gathered}
$$

and 


$$
e_{i j k} \sim N\left(0, \sigma_{e}^{2}\right) .
$$

Using Brute Force Method, the expected mean squares of Equation (1) when factor A is fixed and factor B is random the expected mean square are shown in ANOVA Table 2.

From Table 2, if we interested to test for the expression $H_{0}: \alpha_{1}=\alpha_{2}=\cdots=\alpha_{p}$ there are no obvious denominator for testing for the factor $\mathrm{A}$.

However if we can obtain the expression

$$
M S_{\theta_{1}}=K_{1} \sigma_{\lambda}^{2}+\sigma_{\varepsilon}^{2}
$$

we would have the F-test as

$$
F_{f_{\alpha}, f_{\theta}}=\frac{M S_{\alpha}}{M S_{\theta_{1}}}
$$

where $f_{\alpha}$ and $f_{\theta}$ are the numerator and denominator degrees of freedom respectively

$$
\begin{gathered}
\text { where } K_{1}=\left(\frac{\sum_{i} N_{i}^{-1} \sum_{j} n_{i j}^{2}-N^{-1} \sum_{i j} n_{i j}^{2}}{p-1}\right) \\
K_{2}=N^{-1} \sum_{i j} n_{i j}^{2} \\
K_{3}=\frac{\left(\sum_{i j} n_{i j}-N^{-1} \sum_{i j} n_{i j}^{2}-\sum_{i} N_{i}^{-1} \sum_{j} n_{i j}^{2}\right)}{(p-1)(q-1)} .
\end{gathered}
$$

Using Welch Satterthwaite Equation $f_{\phi}$ is the degree of freedom for the denominator and is given by

$$
f_{\phi}=\frac{\left(M S_{\phi_{1}}\right)^{2}}{(1-\theta)^{2} \frac{\left(M S_{e}\right)^{2}}{f_{e}}+\theta^{2} \frac{\left(M S_{\lambda}\right)^{2}}{f_{\lambda}}}
$$

$M S_{\theta_{1}}$ can be shown to be

$$
(1-\theta) M S_{\varepsilon}+\theta M S_{\lambda}
$$

where

$$
\theta=\frac{K_{1}}{K_{3}}
$$

Table 2. ANOVA table two way unbalanced mixed interactive model when factor A is fixed and factor $\mathrm{B}$ is random.

\begin{tabular}{ccccc}
\hline S.V & d.f & SS & MS & Expected mean square \\
\hline$p$-classes & $p-1$ & $\mathrm{SS}_{\alpha}$ & $\mathrm{MS}_{\alpha}$ & $\frac{\sum_{i} N_{i} \alpha_{i}^{2}}{p-1}+K_{1} \sigma_{\lambda}^{2}+\sigma_{\varepsilon}^{2}$ \\
$q$-classes & $q-1$ & $\mathrm{SS}_{\beta}$ & $\mathrm{MS}_{\beta}$ & $K_{\beta} \sigma_{\beta}^{2}+k_{2} \sigma_{\lambda}^{2}+\sigma_{\varepsilon}^{2}$ \\
$p q$-classes & $(p-1)(q-1)$ & $\mathrm{SS}_{\lambda}$ & $\mathrm{MS}_{\lambda}$ & $k_{3} \sigma_{\lambda}^{2}+\sigma_{\varepsilon}^{2}$ \\
Error & $N-p q$ & $\mathrm{SS}_{\varepsilon}$ & $\mathrm{MS}_{\varepsilon}$ & $\sigma_{\varepsilon}^{2}$ \\
Total & $N-1$ & $\mathrm{SS}_{\mathrm{T}}$ & - & - \\
\hline
\end{tabular}


Statement 1: Equation (37) is an unbiased estimate of Equation (31).

Proof:

We take expectation on Equation (33) to have

$$
\begin{aligned}
& E\left(M S_{\theta_{1}}\right)=E\left[(1-\theta) M S_{\varepsilon}+\theta M S_{\lambda}\right] \\
& =(1-\theta) \sigma_{\varepsilon}^{2}+\theta\left(K_{3} \sigma_{\lambda}^{2}+\sigma_{\varepsilon}^{2}\right) \\
& =\sigma_{\varepsilon}^{2}-\theta \sigma_{\varepsilon}^{2}+\theta K_{3} \sigma_{\lambda}^{2}+\phi \sigma_{\varepsilon}^{2} .
\end{aligned}
$$

But $\theta=\frac{K_{1}}{K_{3}}$

$$
\begin{aligned}
& \therefore E M S_{\theta_{1}}=\sigma_{\varepsilon}^{2}+\frac{K_{1}}{K_{\mathrm{B}}} \cdot \mathrm{K}_{\mathrm{s}} \sigma_{\lambda}^{2} \\
& =K_{1} \sigma_{\lambda}^{2}+\sigma_{\varepsilon}^{2} \text { as required. }
\end{aligned}
$$

Similarly, if we are interested to test for factor $\mathrm{B}$ we have

$$
H_{0}: \sigma_{\beta}^{2}=0
$$

there would be no obvious denominator to test for the above hypothesis. However, if we can obtain the expression

$$
M S_{\theta_{2}}=k_{2} \sigma_{\lambda}^{2}+\sigma_{\varepsilon}^{2} .
$$

We would have the F-test as

$$
F_{f_{\beta}, f_{\theta}}^{\alpha}=\frac{M S_{\beta}}{M S_{\theta_{2}}}
$$

where $f_{\beta}$ and $f_{\theta}$ are the numerator and denominator degrees of freedom respectively.

$f_{\alpha}$ is the degree of freedom for the numerator and $f_{\phi}$ is the degree of freedom for the denominator and is given by

$$
f_{\phi}=\frac{\left(M S_{\phi_{2}}\right)^{2}}{(1-\theta)^{2} \frac{\left(M S_{e}\right)^{2}}{f_{e}}+\theta^{2} \frac{\left(M S_{\lambda}\right)^{2}}{f_{\lambda}}}
$$

where $f_{e}$ and $f_{\lambda}$ are the degrees of freedom for the error components and the interactions respectively.

$M S_{\theta_{2}}$ can be shown to be

$$
\begin{gathered}
(1-\theta) M S_{\varepsilon}+\theta M S_{\lambda} \\
\theta=\frac{K_{2}}{K_{3}}
\end{gathered}
$$

Equation (41) is also an unbiased estimate of Equation (38).

Similarly, if factor A is random, and factor B is fixed, the expected mean square are shown in the ANOVA Table 3.

Where

$$
\begin{gathered}
K_{\alpha}=\frac{\sum_{i} N_{i}\left(\alpha_{i}-\bar{\alpha}\right)^{2}}{p-1} \\
K_{4}=\frac{N^{-1} \sum_{i j} n_{i j}^{2}}{p-1}
\end{gathered}
$$


Table 3. ANOVA table two way unbalanced mixed interactive model when factor A is random and factor B is fixed.

\begin{tabular}{ccccc}
\hline S.V & d.f & SS & MS & Expected mean square \\
\hline$p$-classes & $p-1$ & $\mathrm{SS}_{\alpha}$ & $\mathrm{MS}_{\alpha}$ & $K_{\alpha} \sigma_{\alpha}^{2}+K_{4} \sigma_{\lambda}^{2}+\sigma_{\varepsilon}^{2}$ \\
$q$-classes & $q-1$ & $\mathrm{SS}_{\beta}$ & $\mathrm{MS}_{\beta}$ & $\frac{\sum_{j} N_{j} \beta_{j}^{2}}{q-1}+k_{5} \sigma_{\lambda}^{2}+\sigma_{\varepsilon}^{2}$ \\
$p q$-classes & $(p-1)(q-1)$ & $\mathrm{SS}_{\lambda}$ & $\mathrm{MS}_{\lambda}$ & $k_{3} \sigma_{\lambda}^{2}+\sigma_{\varepsilon}^{2}$ \\
Error & $N-p q$ & $\mathrm{SS}_{\varepsilon}$ & $\mathrm{MS}_{\varepsilon}$ & $\sigma_{\varepsilon}^{2}$ \\
Total & $N-1$ & $\mathrm{SS}_{\mathrm{T}}$ & - & - \\
\hline
\end{tabular}

$$
K_{5}=\frac{\left(\sum_{j} N_{j}^{-1} \sum_{i} n_{i j}^{2}-N^{-1} \sum_{i j} n_{i j}^{2}\right)}{q-1} .
$$

When factor A is random, the hypothesis is given by

$$
H_{0}: \sigma_{\alpha}^{2}=0
$$

and there would be no obvious denominator to test for the above hypothesis. If we can obtain the expression

$$
M S_{\theta_{4}}=k_{4} \sigma_{\lambda}^{2}+\sigma_{\varepsilon}^{2}
$$

the F-test can be shown to be

$$
\begin{gathered}
F_{f_{\alpha}, f_{\theta}}^{\alpha}=\frac{M S_{\alpha}}{M S_{\theta_{4}}} \\
M S_{\theta_{4}}=(1-\theta) M S_{\varepsilon}+\theta M S_{\lambda} \\
\theta=\frac{K_{4}}{K_{3}}
\end{gathered}
$$

where $f_{\alpha}$ and $f_{\theta}$ are the numerator and denominator degrees of freedom respectively.

$$
f_{\phi}=\frac{\left(M S_{\phi_{4}}\right)^{2}}{(1-\theta)^{2} \frac{\left(M S_{e}\right)^{2}}{f_{e}}+\theta^{2} \frac{\left(M S_{\lambda}\right)^{2}}{f_{\lambda}}}
$$

where $f_{e}$ and $f_{\lambda}$ are the degrees of freedom for the error components and the interactions respectively.

Equation (47) is also an unbiased estimate of Equation (45).

Similarly, when factor B is fixed, the hypothesis is given by

$$
H_{0}: \beta_{1}=\beta_{2}=\cdots=\beta_{q}
$$

with no obvious denominator to test for the hypothesis. However, if we can obtain the expression

$$
M S_{\theta_{5}}=k_{5} \sigma_{\lambda}^{2}+\sigma_{\varepsilon}^{2}
$$

the F-test can be shown to be

$$
F_{f_{\beta}, f_{\theta}}^{\alpha}=\frac{M S_{\beta}}{M S_{\theta_{5}}}
$$




$$
\begin{gathered}
M S_{\theta_{5}}=(1-\theta) M S_{\theta}+\theta M S_{\lambda} \\
\theta=\frac{K_{5}}{K_{3}} \\
f_{\phi}=\frac{\left(M S_{\phi_{5}}\right)^{2}}{(1-\theta)^{2} \frac{\left(M S_{e}\right)^{2}}{f_{e}}+\theta^{2} \frac{\left(M S_{\lambda}\right)^{2}}{f_{\lambda}}}
\end{gathered}
$$

where $f_{\beta}$ and $f_{\theta}$ are the numerator and denominator degrees of freedom respectively.

Similarly Equation (51) is also an unbiased estimate of Equation (49).

Finally, the hypothesis for testing for the presence of the interaction is given by

$$
F_{f_{\lambda}, f_{\varepsilon}}^{\alpha}=\frac{M S_{\lambda}}{M S_{\varepsilon}}
$$

where $f_{\lambda}$ and $f_{\varepsilon}$ are the numerator and denominator degrees of freedom respectively.

\section{Conclusions}

Equation (2) contains the functions of the fixed effect which is $\sum_{i=1}^{p} n_{i} \alpha_{i}^{2}-\frac{\left(\sum_{i=1}^{p} n_{i} \alpha_{i}\right)^{2}}{n}$. If we ignore the fixed effects and eliminate them from the model, what remains is a random model for which the F-test can be determined. The second possibility is to assume the fixed effects as random and therefore assume the entire model as random effect models. This is completely unreasonable.

From Table 2 and Table 3, when one factor is fixed, we equate the functions of the fixed effect to zero and obtain an expression to determine the denominator for the F-ratio when the hypothesis is specified. Similarly, when the other factor is random, we equate the functions of the random effect to zero and obtain an expression to determine the denominator for the F-ratio when the hypothesis is specified.

To test for the interaction effect for the mixed effect model, we have

$$
F_{f_{\lambda}, f_{\varepsilon}}^{\alpha}=\frac{M S_{\lambda}}{M S_{\varepsilon}} .
$$

This does not involve obtaining any expression and the degrees of freedom for both the numerator and denominator are integer valued whereas the denominator degrees of freedom for the testing for the main effects are non integer valued.

Instead of assuming both effects to be fixed or both effects to be random to enable researchers on mixed effect unbalanced interactive model analyze their data, we highly recommend our method.

This paper is limited to only an unbalanced two-way mixed effect interactive model and cannot be applied to random or fixed effect model.

\section{Illustrative Example}

Synthetic growth hormone was administered at a clinical research center to growth hormone deficient 18 short children who had not yet reached puberty. The investigator was interested in the effects of a child's gender (factor A) and bone development (factor B) on the rate of growth induced by hormone administration. A child's bone development was classified into one of the three categories: severely depressed, moderately depressed and mildly depressed. Three children were randomly selected for each gender-bone development group. The response variable $(\mathrm{Y})$ of interest was the difference between the growth rate during hormone treatment and the normal growth rate prior to the treatment, expressed in centimeters per month. Four of the 18 children were unable to complete the study leading to unequal treatment sample sizes shown below.

Since factor A is random and factor B is assumed to fixed, we shall make use of the information in Table 4 . 
Table 4. Growth hormone data.

\begin{tabular}{|c|c|c|c|c|c|}
\hline \multirow[t]{2}{*}{ Gender (factor A) } & \multicolumn{3}{|c|}{ Bone development (factor B) } & \multirow[b]{2}{*}{$N_{j}$} & \multirow[b]{2}{*}{$\bar{Y}_{j .}$} \\
\hline & $\begin{array}{c}\text { Severely } \\
\text { depressed }\end{array}$ & $\begin{array}{l}\text { Moderately } \\
\text { depressed }\end{array}$ & $\begin{array}{c}\text { Mildly } \\
\text { depressed }\end{array}$ & & \\
\hline Male (A1) & $\begin{array}{l}1.4 \\
2.4 \\
2.2\end{array}$ & $\begin{array}{l}2.1 \\
1.7\end{array}$ & $\begin{array}{l}0.7 \\
1.1\end{array}$ & 7 & 1.66 \\
\hline Female (A2) & 2.4 & $\begin{array}{l}2.5 \\
1.8 \\
2.0\end{array}$ & $\begin{array}{l}0.5 \\
0.9 \\
1.3\end{array}$ & 7 & 1.63 \\
\hline$N_{j}$ & 4 & 5 & 5 & 14 & \\
\hline $\bar{Y}_{j .}$ & 2.1 & 2.02 & 0.9 & & $\bar{Y}_{Y . .}=1.64$ \\
\hline
\end{tabular}

Source: Netal et al. (1996) Applied Linear Statistical Models [9].

Our hypothesis for factor A shall be

$$
H_{0}: \sigma_{\alpha}^{2}=0
$$

Using Equations (20), (22) and (23) we have

$$
M S_{A}=0.0035 ; M S_{\lambda}=1.242 \text { and } M S_{e}=0.1625 .
$$

Similarly, using Equations (43) and (44)

$$
K_{4}=2.57, K_{5}=1.29 \text { and } K_{3}=1.87 \text {; hence } \theta=\frac{K_{4}}{K_{3}}=1.37 \text {. }
$$

From Equations (47) and (48)

$$
\begin{aligned}
& M S_{\theta_{4}}=1.641 \text { and } f_{\theta}=1.8621 \\
& \therefore F=\frac{M S_{A}}{M S_{\theta_{4}}}=\frac{0.0035}{1.641}=0.00213 \\
& F_{1,2}^{0.05}=18.51 .
\end{aligned}
$$

Our conclusion is that we do not reject the null hypothesis.

Similarly, our hypothesis for factor B shall be

Using Equation (21)

$$
H_{0}: \beta_{1}=\beta_{2}=\beta_{3} \text {. }
$$

From Equations (51) and (52)

$$
M S_{\beta}=2.153 \text {. }
$$

$$
\begin{aligned}
& M S_{\theta_{5}}=0.91 \text { and } f_{\theta}=2.25 \\
& \therefore F=\frac{M S_{\beta}}{M S_{\theta_{5}}}=\frac{2.153}{0.91}=2.37 \\
& F_{2,2}^{0.05}=19.00 .
\end{aligned}
$$

Our conclusion is that we do not reject the null hypothesis.

Finally, to test for the interaction we have

$$
\begin{aligned}
& F=\frac{M S_{\lambda}}{M S_{\varepsilon}}=\frac{1.242}{0.1625}=7.64 \\
& F_{2,8}^{0.05}=4.46 .
\end{aligned}
$$

Our conclusion is therefore that interaction is present. 


\section{References}

[1] Peretz, C., Goren, A., Smid, T. and Kromhout, H. (2002) Application of Mixed-Effect Models for Exposure Assessment. Annals of Occupational Hygiene, 46, 69-77. http://dx.doi.org/10.1093/annhyg/mef009

[2] Edward, F.V. and Randy, L.C. (1992) Mixed-Effect Nonlinear Regression for Unbalanced Repeated Measures. Biometrics, 48, 1-17. http://dx.doi.org/10.2307/2532734

[3] Ofversten, J. (1993) Exact Tests for Variance Components in Unbalanced Mixed Linear Models. Biometrics, 49, 4557. http://dx.doi.org/10.2307/2532601

[4] Khuri, A.I. and Littell, R.C. (1987) Exact Tests for the Main Effects Variance Components in an Unbalanced Random Two-Way Model. Biometrics, 43, 545-560. http://dx.doi.org/10.2307/2531994

[5] Ananda, M.M.A. and Weerahandi, S. (1997) Two-Way ANOVA with Unequal Cell Frequencies and Unequal Variances. Statistica Sinica, 7, 631-646.

[6] Dawn, I. (1995) Analysis of Variance for Unbalanced Data. Marketing, Theory and Practice, 6, 337-343.

[7] Searle, S.R. (1971) Topics in Variance Components Estimation. Biometrics, 27, 1-76. http://dx.doi.org/10.2307/2528928

[8] Eze, F.C. and Chigbu, P.E. (2012) Unbalanced Two-Way Random Model with Integer-Value Degrees of Freedom. Journal of Natural Sciences Research, 2, 100-107.

[9] Neter, J., Kutner, M.H. and Wasserman, W. (1996) Applied Linear Statistical Models. WCB/McGraw-Hill, 696-700. 